\title{
Estilo de apego y mentalización en condenados por delitos sexuales ${ }^{4}$
}

\section{Ricardo Camilo Rueda Mora}

Magister en Psicología Clínica Universidad del Sinú, Montería, Colombia Correo electrónico: camilorueda@unisinu.edu.co

\section{Rosangela Carolina Raleigh Bethencourt}

Psicóloga

Universidad del Sinú, Montería, Colombia Correo electrónico: rosangela.raleigh@gmail.com

\section{Dayana Carolina Causil Montes \\ Psicóloga Universidad del Sinú, Montería, Colombia Correo electrónico: dayanacausil2@gmail.com}

\section{Army Rosa González Tordecilla \\ Psicóloga \\ Universidad del Sinú, Montería, Colombia Correo electrónico: armygt@outlook.com \\ Luis Hernando Chartuny Chimá}

Magíster en Educación Universidad del Sinú, Montería, Colombia Correo electrónico: Ichartuny@gmail.com
Recibido: 03/08/2018

Evaluado: $28 / 08 / 2018$ Aceptado: 24/09/2018

\section{Resumen}

El objetivo del artículo es describir la relación entre el estilo de apego y la mentalización en condenados por delitos sexuales que se encuentran recluidos en el Establecimiento Penitenciario y Carcelario de la ciudad de Montería-Córdoba, Colombia. Fue un estudio de tipo cuantitativo, descriptivo correlacional y de corte transversal, con una población de 96 condenados por delitos sexuales y una muestra de 50 hombres divididos en 6 categorías según el tipo de delito sexual. La media de edad fue 45 años y se evaluaron datos sociodemográficos, estilos de apego con el CaMir-R y mentalización con el RFQ de 8 ítems. La mayoría de los sujetos presentó mayor proporción de "apego inseguro", y dificultades en la mentalización. La dimensión "Autonomía y rencor contra los padres" perteneciente al tipo de apego inseguro fue la única que se correlacionó positivamente con la mentalización.

\section{Palabras claves}

Apego, mentalización, condenados, delito sexual.

4 Para citar este artículo: Rueda, R. C., Raleigh, R. Causil-Montes, D. C., Gonzalez-Tordecilla, A. R., \& Chartuny, L. (2019). Estilo de apego y mentalización en condenados por delitos sexuales. Informes Psicológicos, 19(2), pp. 53-65 http://dx.doi.org/10.18566/infpsic.v19n2a04 


\title{
Attachment style and mentalization in convicts for sexual crimes
}

\begin{abstract}
The objective of the article is to describe the relationship between the style of attachment and the mentalization in convicted of sexual crimes that are confined in the Penitentiary and Prison Establishment of the city of Monteria-Córdoba, Colombia. A total of 96 convicted of sexual crimes participated in this quantitative, descriptive correlational and cross-sectional study. The sample consisted of 50 men divided into 6 categories according to the type of sexual offense, with an average age of 45 . Sociodemographic data, attachment styles with the CaMir-R and mentalization with the 8-item RFQ were evaluated. The majority of subjects presented a greater proportion of "insecure attachment" and difficulties in mentalization. The dimension "Autonomy and resentment against parents", belonging to the type of insecure attachment, was the only one that correlated positively with mentalization.
\end{abstract}

Keywords

Attachment, mentalization, convicted, sexual offense.

\section{Estilo de apego e mentalização em condenados por crimes sexuais}

\section{Resumo}

0 objetivo deste artigo é descrever a relação presente entre 0 estilo de apego e a mentalização em condenados por crimes sexuais que se acham recluídos no Estabelecimento Penitenciário e Carcerário da cidade de Montería-Córdoba, Colômbia. Trata-se de um estudo de tipo quantitativo, descritivo correlacional e de corte transversal, com uma população de 96 condenados por crimes sexuais e uma amostra constituída por 50 homens divididos em 6 categorias de acordo com 0 tipo de crime sexual. A média da idade foi de 45 anos e foram avaliados dados sociodemográficos, estilos de apego com o CaMir-R e mentalização com o RFQ de 8 itens. A maioria dos sujeitos deu mostra de uma maior proporção de "apego inseguro", e dificuldades na mentalização. A dimensão "autonomia e rancor contra os pais" pertencente ao tipo de apego inseguro foi a única que foi correlacionada positivamente com a mentalização.

Palavras chave Apego, mentalização, condenados, crime sexual. 


\section{ntroducción}

La violencia sexual en Colombia es altamente prevalente. Según la Encuesta Nacional de Demografía y Salud (ENDS) aproximadamente $5.7 \%$ de las mujeres entre 15 y 49 años han sufrido violencia de este tipo (Profamilia, 2011) y dicha cifra parece ir en aumento. De acuerdo a las denuncias reportadas por el Instituto $\mathrm{Na}$ cional de Medicina Legal y Ciencias Forenses (Canesto, 2017), se registraron 23.798 casos de violencia sexual durante el año 2017, es decir, un incremento del 11.2\% respecto al 2016.

En consecuencia, esto constituye un problema social y de salud pública, por lo que se han derivado diferentes formas de abordarlo, en su mayoría enfocadas en el tratamiento de las víctimas de abuso sexual en la infancia y la adultez (Echeburúa \& Guerricaechevarría, 2011; Sarasua, Zubizarreta, de Corral, \& Echeburúa, 2013), mientras que usualmente no se encontraba información académica acerca del agresor en la misma proporción que de la víctima; es por ello que se están llevando a cabo investigaciones dirigidas al agresor (Sorrentino, Brown, Berard, \& Peretti, 2018). Acerca de éste, se han encontrado rasgos como: baja autoestima, déficit en habilidades verbales y asertividad (Rodríguez, 2003), dificultad en la regulación afectiva (Gunst, Watson, Desmet, \& Willemsen, 2017) y en la teoría de la mente (Castellino, Bosco, Marshall, Marshall, \& Veglia, 2011) siendo este último, un concepto equivalente a la función reflexiva o mentalización.

La función reflexiva es la habilidad de dar una interpretación convincente a la conducta propia y de otros (Fonagy, 2002); esta capacidad puede ser un determinante de la regulación afectiva y emerge de las relaciones tempranas de apego (Fonagy \& Bateman, 2016). Dentro de las fallas presentadas al momento de mentalizar se encuentran la hipermentalización, donde los sujetos asumen explicaciones a las conductas que tienen otras personas a partir de estados emocionales sin un asentamiento en la realidad fáctica; y la hipomentalización que se refiere a la dificultad para considerar la complejidad de los procesos mentales propios y de los otros haciendo difícil la comprensión de estos, impidiendo entender cómo las acciones afectan o le ocasionan daño a otra persona (Fonagy, 2002), lo que hace pensar en la relevancia de la capacidad de mentalización en el funcionamiento de los agresores sexuales.

Esto se respalda con resultados de investigaciones sobre otros tipos de conducta violenta, en donde se ha encontrado un deterioro en la capacidad para mentalizar en delincuentes con trastorno antisocial de la personalidad (Newbury-Helps, Feigenbaum, \& Fonagy, 2017), deficiencias en la Teoría de la Mente (ToM) en población colombiana con trastorno disocial de la conducta (Gómez, Arango, \& Molina, 2010) y en población normal con conductas violentas vinculadas con el conflicto armado (Gómez \& Molina, 2013).

De igual manera, se establece una relación entre el apego adulto y la conducta sexual violenta. El apego es un sistema de protección y supervivencia en el que el niño, ante situaciones de estrés o peligro, busca apoyo en la figura materna o cuidador principal. Las diferentes experiencias en el marco de esta relación crean un patrón de conducta que se clasifica como seguro o inseguro; dentro del patrón de 
apego de tipo inseguro se describen dos subtipos de conducta: el apego ansioso y el apego evitativo (Bowlby, 1998). Los sujetos con apego seguro se caracterizan por confiar en sí mismos y los otros, así como establecer relaciones interpersonales satisfactorias y dar importancia a la estructura familiar. Mientras que las personas con un apego de tipo ansioso son descritas como preocupadas constantemente en relación a la pérdida o abandono de los familiares y mostrando dificultades para actuar de forma independiente. Por otro lado, quienes muestran apego evitativo desarrollan una pseudoindependencia marcada por relaciones restrictivas con los otros (Lacasa \& Muela, 2014).

Martin \& Tardif (2013) han encontrado déficit en la capacidad para intimar en agresores sexuales marcado por un estilo inseguro de apego, vinculada a la relación con la figura de apego en la infancia y que permanecerá relativamente estable en la edad adulta (Pimentel \& Santelices, 2017; Smallbone \& Dadds, 1998). El apego es un factor fundamental en el desarrollo de todas sus dimensiones, de tal manera que las dificultades en el marco de estas relaciones pueden traer consecuencias en la adultez afectando la construcción del sujeto y la conducta social (Calle, 2012). De hecho, las teorías freudianas referentes a la sexualidad infantil explican cómo las perversiones sexuales se originan durante la infancia (Bowlby, 1998), al igual que las expectativas en cuanto a la naturaleza de las interacciones y la función reflexiva (Fonagy, 2002).

En este sentido, tomando en cuenta el rol que juega la mentalización y el apego en las conductas violentas, se puede inferir que los agresores sexuales presentan dificultades en estas variables. Por ello, la presente investigación estableció como objetivo describir la relación entre la mentalización y el apego en una población condenada por delitos sexuales en la ciudad de Montería-Córdoba, Colombia, con el fin de obtener mayor comprensión acerca del funcionamiento psicológico y emocional de estos sujetos y generar modelos de intervención y prevención que tengan en cuenta estas características.

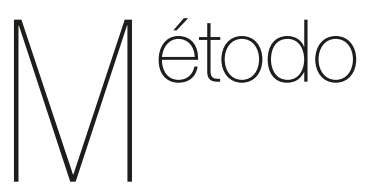

\section{Participantes}

El establecimiento penitenciario y carcelario de la ciudad de Montería-Córdoba, Colombia, cuenta con una población de 1.783 internos recluidos, de los cuales 96 son población condenada por delitos sexuales. Se realizó un muestreo de tipo intencional conformado por 50 sujetos internos condenados por delito sexual que cumplieron con los criterios de inclusión. Dichos criterios fueron los siguientes: estar condenados por delitos sexuales, estar ubicados en edades entre 18 y 70 años, saber leer y escribir y no tener un trastorno mental diagnosticado.

\section{Instrumentos}

\section{Cuestionario de datos sociodemográficos}

Se llevó a cabo un cuestionario para indagar las variables edad, estado civil, nivel socioeconómico, número de hijos, nivel de escolaridad, tipo de familia a la 
que pertenece, conformación familiar, relaciones afectivas con padres y hermanos, tiempo de condena en meses y tipo de delito sexual.

\section{Cuestionario de apego Camir-R}

(Balluerka, Lacasa, Gorostiaga, Muela, \& Pierrehumbert, 2011). Se trata de un instrumento de auto reporte para medir la concepción respecto al funcionamiento de la familia y la representación del tipo de apego y las figuras protectoras en la adolescencia y la edad adulta. El cuestionario se encuentra compuesto por 32 reactivos que se responden con una escala Likert de 5 puntos, partiendo de Totalmente en desacuerdo (1) a totalmente de acuerdo (5) (Lacasa \& Muela, 2014).

cuestionario está estructurado a partir de siete dimensiones, cinco de las cuales se refieren a la representación del tipo de apego, éstas son: (a) seguridad (disponibilidad y apoyo de las figuras de apego), (b) preocupación familiar, (c) interferencia de los padres, (d) autosuficiencia y rencor contra los padres, y (e) traumatismo infantil. Las dos dimensiones restantes se dirigen a la representación de la estructura familiar y son: (a) valor de la autoridad de los padres, y (b) permisividad parental (Lacasa \& Muela, 2014). La validación realizada por Balluerka et al. (2011) reporta validez convergente y una consistencia interna de .60 a .85 .

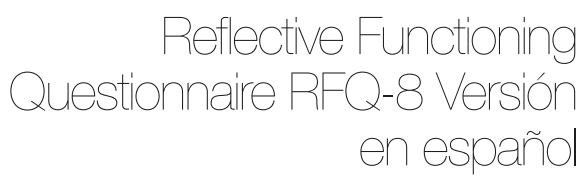

(Fonagy et al., 2016). El instrumento para la evaluación de la mentalización consta de 8 ítems con dos sub escalas, una que evidencia la hipermentalización (RFQ_C), la cual representa la certeza acerca de los estados mentales y otra la hipomentalización (RFQ_U) u opacidad de los estados mentales. Ambas escalas siguen una puntuación que va del 1 al 7 y se recodifican como 3,2,1,0,0,0,0 en el primer caso y en el segundo caso $0,0,0,0,1,2,3$. Los puntajes altos en las dos escalas reflejan las dificultades en la función reflexiva (Fonagy et al., 2016). El Alfa de Cronbach de RFQ_U fue de .77 y en la de RFQ_C fue de .65; adicionalmente, en la investigación original se aplicó el método test-retest después de un período de tres semanas, resultando una excelente correlación de .84 y .85, respectivamente (Fonagy et al., 2016).

\section{Procedimiento}

Se solicitó el permiso para el ingreso al establecimiento para la aplicación. Se obtuvo el consentimiento informado, posteriormente los sujetos fueron llamados de forma individual en una única sesión donde se suministró el Cuestionario de Variables Sociodemográficas, el CaMir y el RFQ. Esto se realizó en los meses de mayo y junio de 2017, dos (2) días a la semana. Los participantes recibieron un incentivo previamente aprobado por la institución. El tratamiento estadístico de la información se realizó mediante el programa SPSS versión 23, con el cual se realizó un análisis descriptivo de las variables sociodemográficas, así como de los datos obtenidos en las pruebas. También se utilizó el coeficiente de correlación de Pearson para determinar las relaciones entre las dimensiones de las variables de Apego y Mentalización. Por último, se contrastó la clasificación cualitativa de los puntajes de las pruebas con los datos sociodemográficos, usando la fórmula de Chi-cuadrado. 


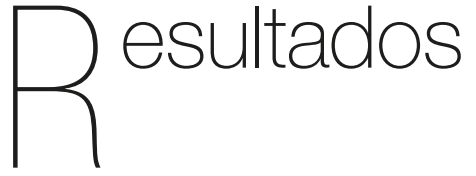

La Tabla 1 muestra una descripción de los datos sociodemográficos obtenidos. Es importante destacar que el 84\% de los participantes tienen un bajo nivel de escolaridad y que en su mayoría pertenecen al estrato socioeconómico más bajo y el $98 \%$ manifestaron realizar algún tipo de trabajo antes de ser condenados. En cuanto a las relaciones personales, el $60 \%$ de los sujetos de la muestra manifestaron no tener un vínculo con una pareja estable, y el $68 \%$ pertenecieron a familias de tipo monoparental o de padres separados. En el estudio se encontró además que el $76 \%$ de los condenados cometieron delito sexual con menores de 14 años.

Tabla 1.

Características sociodemográficas de la muestra $(n=50)$.

\begin{tabular}{|c|c|c|c|c|c|}
\hline & $\mathrm{n}$ & $\%$ & & $\mathrm{n}$ & $\%$ \\
\hline Edad & & & Estado Civil & & \\
\hline Media (D.T.) & 45.2 & 13.5 & Casado & 6 & 12 \\
\hline Min-Máx & 22 & 70 & Soltero & 21 & 42 \\
\hline Nivel de escolaridad & & & En unión libre & 14 & 28 \\
\hline Analfabeto & 2 & 4 & Separado & 9 & 18 \\
\hline Básica primaria incompleta & 22 & 44 & Tipo de delito sexual & & \\
\hline Básica primaria completa & 4 & 8 & Acceso carnal violento & 6 & 12 \\
\hline Básica secundaria incompleta & 14 & 28 & Acto sexual violento & 1 & 2 \\
\hline Básica secundaria completa & 5 & 10 & $\begin{array}{l}\text { Acceso carnal o acto sexual en persona } \\
\text { puesta en incapacidad de resistir }\end{array}$ & 2 & 4 \\
\hline Tecnológico/técnico incompleta & 1 & 2 & Acceso carnal abusivo con menor de 14 años & 23 & 46 \\
\hline Tecnológico/técnico & 1 & 2 & Acto sexual con menor de 14 años & 15 & 30 \\
\hline Universitario completo & 1 & 2 & $\begin{array}{l}\text { Acceso carnal o acto sexual abusivo } \\
\text { con incapacidad para resistir }\end{array}$ & 3 & 6 \\
\hline Actividad previa al delito & & & Tipo de familia & & \\
\hline Trabajaba & 49 & 98 & Nuclear & 12 & 24 \\
\hline \multirow[t]{3}{*}{ Estudiaba } & 1 & 2 & Extensa & 4 & 8 \\
\hline & & & Monoparental & 19 & 38 \\
\hline & & & Padres separados & 15 & 30 \\
\hline
\end{tabular}

En las puntuaciones arrojadas por el CaMir-R se puede apreciar que la mayoría de los participantes $(n=39)$ tenía un tipo de apego calificado como inseguro (18 preocupado, 21 evitativo). En relación al RFQ, el $74 \%$ de la muestra presenta dificultades en el proceso de mentalización, de los cuales un 28\% tiene hipermentalización mientras que el $42 \%$ presenta hipomentalización, y un $4 \%$ evidencia los dos tipos de dificultades. Cabe destacar que dentro de los sujetos que presentaron fallas, el $81.1 \%$ también tenían un tipo de apego inseguro.

En cuanto al tipo de apego y tipo de delito, se encontró que el $46 \%$ de los privados de libertad cometieron delitos por acceso carnal abusivo con menores de 
edad; en segundo lugar, se encuentran aquellos que cometieron actos sexuales con menores de 14 años. Por su parte, el 69.5\% de los sujetos que habían cometido delito sexual del primer tipo mencionado, presentaban además apego inseguro, mientras que, para el segundo tipo, en el 93.3\% se encontraron dificultades en el apego.

Respecto a las variables función reflexiva y tipo de delito sexual, se observó que un $69.5 \%$ de quienes cometieron acceso carnal abusivo a menores de edad, también presentaban fallas en la mentalización. De igual manera, el 86.6\% de los privados que tuvieron actos sexuales con menores de 14 años, evidenciaron fallas en la mentalización. En resumen, todo lo anterior apunta a que los sujetos condenados por delitos sexuales que tienen un tipo de apego inseguro y/o alguna dificultad en el proceso de mentalización tuvieron tendencia a cometer delitos sexuales con victimas menores de edad.

Por otro lado, se aplicó el coeficiente de correlación de Pearson a las puntuaciones de apego y mentalización. Se observó que las puntuaciones de hipomentalización y de hipermentalización correlacionan únicamente con una dimensión del CaMir-R, denominada Autosuficiencia y rencor contra los padres; específicamente la hipomentalización mostró una relación positiva con este tipo de apego y la hipermentalización una relación negativa (ver Tabla 2). Esto quiere decir que cuanto mayor rencor existe hacia los padres, hay mayor incertidumbre al momento de realizar hipótesis acerca del estado mental propio y del otro (hipomentalización).

Tabla 2.

Correlación de Pearson para mentalización y tipo de apego.

\begin{tabular}{lcc}
\hline Dimensiones del CaMir-R & $\begin{array}{c}\text { Promedio } \\
\text { de C }\end{array}$ & $\begin{array}{c}\text { Promedio } \\
\text { de U }\end{array}$ \\
\hline Seguridad & -.018 & -.138 \\
Preocupación familiar & -.12 & -.01 \\
Indiferencia de los padres & .06 & .048 \\
$\begin{array}{l}\text { Valor de la autoridad } \\
\text { de los padres }\end{array}$ & -.128 & -.001 \\
$\begin{array}{l}\text { Permisividad parental } \\
\text { Autosuficiencia y rencor }\end{array}$ & .072 & .126 \\
contra los padres & $-.283^{*}$ & $.346^{*}$ \\
Traumatismo infantil & -.14 & .255 \\
\hline
\end{tabular}

Adicionalmente, se determinó la relación entre el tipo de apego, fallas en mentalización y las siguientes variables sociodemográficas: tipo de familia, tipo de delito, conformación familiar y estrato socioeconómico. Para esto se aplicó el estadístico chi-cuadrado y se observó que el tipo de familia afectó de manera significativa al tipo de apego $(\chi 2(2)=9.09, \mathrm{p}=$ .029), hallándose proporciones más altas en familias monoparentales y de padres separados con respecto al tipo de apego inseguro (ver Tabla 3).

Tabla 3.

Tabla cruzada entre variables nominales tipo de familia y tipo de apego.

\begin{tabular}{|c|c|c|c|c|c|c|}
\hline & & \multicolumn{5}{|c|}{ Tipo de familia } \\
\hline & & Nuclear (\%) & Extensa (\%) & Monoparental (\%) & Padres separados (\%) & Total \\
\hline \multirow{2}{*}{ Tipo de apego } & Inseguro & 25.6 & 2.6 & 35.9 & 35.9 & 39 \\
\hline & Seguro & 18.2 & 27.3 & 45.5 & 9.1 & 11 \\
\hline Total & & 24.0 & 8.0 & 38.0 & 30.0 & 50 \\
\hline
\end{tabular}


De igual forma, se observaron diferencias significativas entre tipo de apego y tipo de delito sexual $X^{2}=11.33, p=$ .045); especialmente se manifestó mayor proporción de apego inseguro en actos sexuales con menores de 14 años. Por otro lado, también se encontraron diferencias entre tipo de familia y tipo de apego $\left.X^{2}=11.33, p=.045\right)$ (ver Tabla 4).

Tabla 4.

Tabla cruzada entre variables nominales tipo de apego y tipo de delito.

\begin{tabular}{|c|c|c|c|c|}
\hline & & \multicolumn{2}{|c|}{ Tipo de familia } & \multirow{2}{*}{ Tota } \\
\hline & & Inseguro (\%) & Seguro (\%) & \\
\hline \multirow{6}{*}{$\begin{array}{l}\text { Tipos de delito } \\
\text { sexual }\end{array}$} & Acceso carnal violento & 83.3 & 16.7 & 6 \\
\hline & Acto sexual violento & 100.0 & & 1 \\
\hline & $\begin{array}{l}\text { Acceso carnal o acto sexual en persona } \\
\text { puesta en incapacidad de resistir }\end{array}$ & & 100.0 & 2 \\
\hline & Acceso carnal abusivo con menor de 14 años & 69.6 & 30.4 & 23 \\
\hline & Actos sexuales con menores de 14 años & 93.3 & 6.7 & 15 \\
\hline & $\begin{array}{l}\text { Acceso carnal o acto sexual con persona } \\
\text { con incapacidad de resistir }\end{array}$ & 100.0 & & 3 \\
\hline \multirow{2}{*}{ Total } & & 39 & 11 & 50 \\
\hline & & 78.0 & 22.0 & \\
\hline
\end{tabular}

Por último, las fallas en la mentalización presentaron diferencias significativas respecto al tipo de familia. Se evidencia que aquellos participantes pertenecientes a familias monoparentales muestran mayor proporción de hipomentalización y los que tienen padres separados, mayor proporción de hipermentalización (ver Tabla 5).

Tabla 5.

Tabla cruzada entre variables nominales tipo de familia y fallas en la mentalización.

\begin{tabular}{clccccc}
\hline & \multicolumn{7}{c}{ Tipo de familia } \\
\hline \multirow{3}{*}{$\begin{array}{c}\text { Fallas en } \\
\text { Mentalización }\end{array}$} & Suclear (\%) & Extensa (\%) & $\begin{array}{c}\text { Monoparental } \\
\text { (\%) }\end{array}$ & $\begin{array}{c}\text { Padres } \\
\text { separados (\%) }\end{array}$ & Total \\
\cline { 2 - 7 } & Hipermentalización & 14.3 & 7.1 & 28.6 & 50.0 & 13 \\
\cline { 2 - 7 } & Hipomentalización & 23.8 & 4.8 & 47.6 & 23.1 & 21 \\
\cline { 2 - 7 } & Falla en ambas & 0.0 & 100.0 & 0.0 & 0.0 & 2 \\
\hline Total & 24.0 & 8.0 & 38.0 & 30.0 & 50 \\
\hline
\end{tabular}




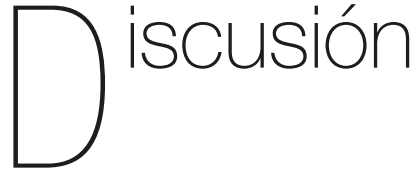

A partir de la información recolectada en el estudio se determinó el tipo de apego y la función reflexiva de la población condenada por delitos sexuales, en su mayoría presentando un estilo de apego "inseguro", lo cual coincide con la literatura relacionada con los agresores sexuales (Abracen, Looman, Di Fazio, Kelly, \& Stirpe, 2006; Marsa et al., 2004; Smallbone \& Dadds, 1998). De igual forma, se pudo identificar que estos sujetos, por lo general, tienen por lo menos una dificultad en su proceso de mentalización siendo en este caso más frecuente la hipomentalización.

Respondiendo a lo planteado al inicio de esta investigación, se puede observar que las dificultades en el apego y mentalización son frecuentes en sujetos condenados por delitos sexuales; además permitió describir el contexto sociodemográfico en el que se encuentran, determinando que un número significativo de esta población pertenece a estratos socioeconómicos bajos y tienen un bajo nivel de escolaridad. Sin embargo, tal como lo señaló Bejarano (2016) en su investigación, los abusadores viven en diferentes contextos, ya sean rurales o urbanos, tienen diferentes niveles de educación, religiones diversas, así como distintos estados civiles y orientaciones sexuales.

Se pudo evidenciar en los resultados obtenidos que el $71.1 \%$ de los agresores con apego inseguro pertenecieron a familias monoparentales y de padres separados. Esto puede estar relacionado con la importancia de las experiencias tempranas del infante en la configuración del sujeto y la formación de vínculos afectivos que proveen un sentimiento de seguridad, tal como lo afirma Bowlby (1998): todo esto se verá reflejado en la vida adulta en variables subjetivas y relaciones sociales satisfactorias. Por ejemplo, en una población similar se midieron autoestima y estrategias de afrontamiento, resultando bajos niveles de autoestima desde la relación con su familia, los cuales, a su vez, se encuentran relacionados con evitación emocional (Larrota, Sánchez, \& Sánchez, 2016).

En cuanto a los tipos de apego, se identificó además que los participantes tenían mayormente un tipo de apego evitativo o preocupado, aunque no se logró describir de forma específica para cada tipo de apego la falla correspondiente en el proceso de mentalización. Resultados en consonancia con la literatura e investigaciones en el tema que encuentran que la mayoría de ofensores sexuales presentan un tipo de apego inseguro (Iffland, Berner, \& Briken, 2013; Marsa et al. 2004; Martin \& Tardif, 2013).

Respecto a la función reflexiva, se evidenció que una gran proporción de los condenados que participaron en el estudio presentan dificultades en el proceso de mentalización, lo que apoya la investigación realizada por Gómez y Molina (2013) quienes refieren que estas fallas en la mentalización pueden incidir en la conducta del ser humano y llevarlos a actuar de una forma que puede afectarlos a sí mismos o a los que se encuentran a su alrededor. En este sentido, se puede pensar entonces 
que la función reflexiva tiene influencia sobre la conducta humana, ya que un desarrollo apropiado de esta capacidad permitiría en gran medida controlar de manera adecuada las emociones y así prevenir el desarrollo de conductas que pueden conllevar a que se cometan este tipo de delitos. Así mismo, los hallazgos de Newbury-Helps et al. (2017) encuentran un deterioro significativo en ofensores con y sin trastorno antisocial de la personalidad.

Se encontró que la mentalización se relaciona directamente con el apego en una sola dimensión (autosuficiencia/rencor contra los padres), la cual es un indicador de apego inseguro de tipo evitativo. En otra investigación, se encontró una correlación baja entre mentalización y estilo de apego inseguro de tipo ansioso (Marszal \& Janczak, 2017), lo que difiere parcialmente con esta investigación, ya que ambas relaciones corresponden a subtipos diferentes de estilo de apego inseguro. En contraste, en otra investigación se encuentra que mentalización solo se relaciona con apego evitativo (Bączkowski \& Cierpiałkowska, 2015), coincidiendo con los hallazgos de esta investigación.

Al respecto, Fonagy (2002) refiere que la mentalización es una variable independiente al apego, a pesar de que para su desarrollo es indispensable contar con unas figuras adecuadas que tengan capacidad para mentalizar. Es por ello que los padres que no poseen la capacidad de mentalizar tampoco pueden favorecer el desarrollo de esta función en sus hijos (Fonagy \& Bateman, 2016). La experiencia de una relación de apego adecuada en la infancia permite el establecimiento de un sistema de representaciones de las experiencias que permite comprender estados mentales de sí mismo y los otros, es decir, mentalizar (McGauley, Yakeley, Williams, \& Bateman, 2011).

En este sentido, la relación entre mentalización y el apego inseguro de tipo evitativo (determinado por la dimensión autosuficiencia y rencor contra los padres) se explica por el rechazo hacia los sentimientos de dependencia que este subtipo de apego presenta. En este tipo de funcionamiento, las representaciones mentales buscan desactivar el sistema de apego (Lacasa \& Muela, 2014), de ahí que se presente una tendencia a hipomentalizar como defensa frente al sentimiento de rechazo e incomprensión por parte de los cuidadores. Dicho esto, la hipomentalización sería una consecuencia de utilizar estas estrategias para protegerse de los sentimientos negativos provocadas por las figuras de apego, tal y como se explica en McGauley et al. (2011). Cabe destacar que, según los resultados de la presente investigación, también se puede observar cómo los sujetos que provienen de familias monoparentales y de padres separados, tienden a desarrollar hipomentalización, lo que puede deberse a la ausencia psíquica de las figuras de apego en estas circunstancias.

Finalmente, es imprescindible a partir de la Psicología continuar investigando la relación entre estas variables, a fin de crear un modelo de intervención efectivo que tenga en cuenta el tipo de apego y que además permita fortalecer la mentalización, con el objetivo de prevenir la conducta de los internos condenados 
por delitos sexuales, ya que claramente estas dos variables se encuentran afectadas en esta población.

De igual manera, es importante crear modelos preventivos que favorezcan el desarrollo de vínculos adecuados y fortalezcan la capacidad de mentalización. Esto, traerá como consecuencia un aumento en la regulación afectiva, el control de impulsos y, sobre todo, en habilidades de cognición social como la empatía; haciendo más difícil que se presenten este tipo de conductas abusivas y violentas, en donde no hay un respeto por el otro en su diferencia.

Asimismo, es fundamental seguir investigando y profundizando acerca de este tema con el fin de poder comprender mejor los factores y variables asociadas en el desarrollo del comportamiento de estos sujetos, que parten desde la infancia, con el fin de encontrar la manera de intervenir tempranamente para evitar que se sigan presentando más problemáticas de este tipo. Por último, se debe tener en cuenta que una limitación del estudio fue el tamaño reducido de la muestra, así como que no se realizó una evaluación psicopatológica de los sujetos y se excluyó de la muestra los que previamente tenían un diagnóstico psiquiátrico, lo que podría afectar los resultados debido a características estructurales de los ofensores sexuales. De igual manera, los instrumentos no se encuentran estandarizados en población colombiana, por lo que no se pueden generalizar los resultados. Este estudio representa un primer acercamiento a las variables de apego y mentalización en población condenada por delitos sexuales.

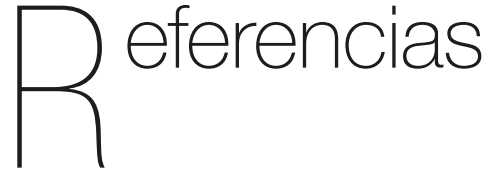

Abracen, J., Looman, J., Di Fazio, R., Kelly, T., \& Stirpe, T. (2006). Patterns of attachment and alcohol abuse in sexual and violent non-sexual offenders. Journal of Sexual Aggression, 12(1), 19-30, doi: https://doi. org/10.1080/13552600600722963

Balluerka, N., Lacasa, F., Gorostiaga, A., Muela, A., \& Pierrehumbert, B. (2011). Versión reducida del cuestionario CaMir-R (CAMIR-R) para la evaluación del apego. Psicothema, 23(3), 486-494.

Bejarano, G. (2016). Sanción penal, efectos carcelarios y reincidencia en delitos sexuales. Revista de Investigación Psicológica, 16, 87-101.

Bowlby, J. (1998). El apego y la perdida - I. El apego. Barcelona: Paidós.

Bączkowski, B., \& Cierpiałkowska, L. (2015). Mentalization within close relationships: The role of specific attachment style. Polish Psychological Bulletin, 46(2), 285-299. doi: 10.1515/ppb-2015-0035

Calle, D. A. (2012). Apego, desarrollo y resiliencia. Informes Psicológicos, 12(1), 25-40.

Canesto, D. (2017). Exámenes medicolegales por presunto delito sexual. Colombia, año 2017. Forensis-Datos para la vida, 19(1), 301-347. Recuperado de http://www.medicinalegal.gov. co/documents/20143/262076/\%20 Forensis+2017+pdf+interactivo.pdf/ e3786e81-8718-b8d5-2731-55758c8ac7ff 
Castellino, N., Bosco, F., Marshall, W., Marshall, L., \& Veglia, F. (2011). Mindreading abilities in sexual offenders: An analysis of theory of mind processes. Consciousness and Cognition, 20(4), 1612-1624.

Echeburúa, E., \& Guerricaechevarría, C. (2011). Tratamiento psicológico de las víctimas de abuso sexual infantil intrafamiliar: un enfoque integrador. Psicología Conductual, 19(2), 469-486.

Fonagy, P. (2002). Affect regulation, mentalization and development of the self. New York: Other Editor.

Fonagy, P., \& Bateman, A. (2016). Tratamiento basado en la mentalización para trastornos de la personalidad. Una guía práctica. España: Editorial Desclée de Brouwer.

Fonagy, P., Luiten, P., Moulton-Perkins, A. Lee, Y.-W., Warren, F., \& Howard, S. (2016). Development and validation of a self-report measure of mentalizing: The Reflective Functioning Questionnaire. PLOS ONE, 11(7), e0158678. doi:10.1371/journal.pone

Gómez, M., \& Molina, D. (2013). Teoría de la mente en un grupo de personas vinculadas al conflicto armado y en proceso de resocialización. Revista Colombiana de Ciencias Sociales, 4, 224-257.

Gómez, M., Arango, O., \& Molina, D. (2010). Características de la teoría de la mente en el trastorno disocial de la conducta. Psicología desde el Caribe, 26, 103-118.

Gunst, E., Watson, J., Desmet, M., \& Willemsen, J. (2017). Affect regulation as a factor in sex offenders. Aggression And Violent Behavior, 37, 210-219.
Iffland, J. A., Berner, W., \& Briken, P. (2013). Relationship factors in sex offenders couples. A pilotstudyinanoutpatient setting. Journal of Sex \& Marital Therapy, 40(6), 529540. doi: 10.1080/0092623X.2013.788108

Lacasa, F., \& Muela, A. (2014). Guía para la aplicación e interpretación del cuestionario de apego CaMir-R. Revista de Psicopatología y Salud Mental del Niño y del Adolescente, 24, 83-93.

Larrota, R., Sánchez, L. \& Sánchez, J. (2016). Niveles de autoestima y uso de estrategias de afrontamiento en un grupo de personas privadas de libertad en un centro de reclusión de la ciudad de Bucaramanga/ Colombia. Informes Psicológicos, 16(1) 51-64.

Marsa, F., O'Reilly, G., Carr, A., Murphy, P., O'Sullivan, M., Cotter, A., \& Hevey, D. (2004). Attachment styles and psychological profiles of child sex offenders in Ireland. Journal of Interpersonal Violence, 19(2), 228-251. doi: 10.1177/0886260503260328

Marszał, M., \& Jańczak, A. (2017). Emotion dysregulation, mentalization and romantic attachment in the nonclinical adolescent female sample. Current Psychology, 1-11, doi: https://doi.org/10.1007/ s12144-017-9573-0

Martin, G. M., \& Tardif, M. (2013). Examining sex offenders' intimacy deficits: their nature and their influence on sexually abusive behaviours. Journal of Sexual Aggression, 21(2), 158-178. doi: 10.1080/13552600.2013.849768

McGauley, G., Yakeley, J., Williams, A., \& Bateman, A. (2011). Attachment, mentalization and antisocial personality disorder: The possible contribution of 
mentalization-based treatment. European Journal of Psychotherapy and Counselling, 13(4), 371-393.

Newbury-Helps, J., Feigenbaum, J., \& Fonagy, P. (2017). Offenders with antisocial personality disorder display more impairments in mentalizing. Journal of Personality Disorders, 31(2), 232-255.

Pimentel, V., \& Santelices, M. (2017). Apego adulto y mentalización en hombres que han ejercido violencia hacia su pareja. Psykhe, 26(2), 1-16. doi: http://dx.doi.org/ 10.7764/psykhe.26.2.915

Profamilia. (2011). Encuesta nacional de demografía y salud 2010. Recuperado de https://dhsprogram.com/pubs/pdf/fr246/ fr246.pdf

Rodríguez, L. (2003). Intervención interdisciplinaria en casos de abuso sexual infantil. Universitas Psychologica, 2(1), 57-70.
Sarasua, B., Zubizarreta, I., de Corral, P., \& Echeburúa, E. (2013). Tratamiento psicológico de mujeres adultas víctimas de abuso sexual en la infancia: resultados a largo plazo. Anales de Psicología, 29(1), 29-37.

Smallbone, S., \& Dadds, M. (1998). Childhood attachment and adult attachment in incarcerated adult male sex offenders. Journal of Interpersonal Violence, 13(5), 555-573.

Sorrentino, R., Brown, A., Berard, B., \& Peretti, K. (2018). Sex offenders: general information and treatment. Psychiatric Annals, 48(2), 120-128. doi: https://doi. org/10.3928/00485713-20171220-01 